Gestión y Estrategia

Gestão e Estratégia

Management and Strategy
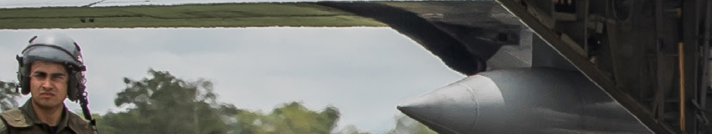

FAC

1005
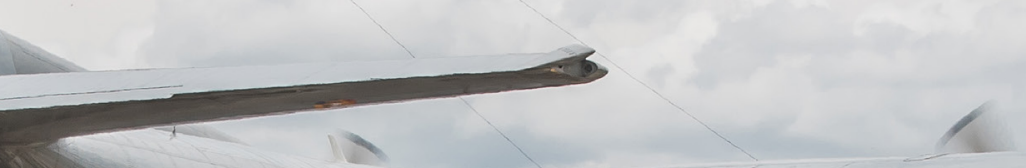

\title{
Fuerza Aérea Colombiana: en la ruta estratégica del futuro Responsable y SOStenible*
}

Força Aérea Colombiana: Na Rota Estratégica o Futuro Responsável e Sustentável **

Colombian Air Force: On the Strategic Route of the Responsible and Sustainable Future ${ }^{* * *}$

Bibiana Enciso Tarquino ${ }^{a}$

Fuerza Aérea Colombiana. Bogotá, Colombia

\section{CIENCIA Y PODER AÉREO}

ISSN 1909-7050 / E- ISSN 2389-9468 / Volumen 12/ Enero-Diciembre de 2017/ Colombia/ Pp. 176-190 Recibido: 02/08/2016

Aprobado: 02/12/2016

Doi: http://dx.doi.org/10.18667/cienciaypoderaereo.570 


\section{Para citar este artículo:}

Enciso, B. (2017). Fuerza Aérea Colombiana: en la ruta estratégica del futuro responsable y sostenible. Ciencia y Poder Aéreo, 12, 176-190. Doi: http://dx.doi.org/10.18667/ cienciaypoderaereo.570

"Artículo de revisión derivado de la investigación presentada como trabajo de grado para optar al título de Magíster en Derechos Humanos y Derecho Internacional de los Conflictos Armados de la Escuela Superior de Guerra. Es producto del proyecto de investigación «Derecho operacional como instrumento eficaz para la protección de la población civil y la seguridad jurídica institucional como individual en Colombia», vinculado al grupo de investigación DDHH, DICA y Justicia, inscrito en Colciencias y avalado por la Escuela Superior de Guerra. Bogotá, Colombia.

*Artigo da revisão derivado da investigação apresentado como tese de graduação para obter o título do Mestrado em Direitos Humanos e Direito Internacional dos Conflitos Armados da Escola Supeior da Guerra, sendo resultado do Projeto de investigação "Direito Operacional como um instrumento efetivo para a proteção dos civis e da segurança jurídica institucional como indivídual na Colômbia" ligado ao grupo de investigação "DDHH, DICA e Justiça"; matriculado no COLCIENCIAS, aprobado por Escola Supeior da Guerra. Bogotá, Colombia.

${ }^{* * *}$ Review article derived from the research submitted as degree work to opt for the Human Rights and International law of armed conflict Master's degree from the War College. It is a product of "Operational Law as an Effective Instrument for the Protection of the Civilian Population and Institutional Legal Security as an Individual in Colombia" project, bounded at the "Human rights, International Law of Armed Conflict and Justice" research group, enrolled at COLCIENCIAS, endorsed by the War College. Bogotá, Colombia.

a Oficial de la Fuerza Aérea Colombiana en el grado de Capitán. Abogada de la Jefatura Jurídica y Derechos Humanos. Correo electrónico: bibiana.enciso@fac.mil.co
Resumen: el presente artículo aborda la realidad de la Responsabilidad Social (RS) al interior de la Fuerza Aérea Colombiana a partir del examen de la estrategia, el modelo y las prácticas de sostenibilidad emprendidas por la Institución que, en sus operaciones aéreas y demás capacidades distintivas o habilidades especiales, define y aplica unas políticas de comando para promoción de la transparencia y la equidad, entre otros valores. Esto mediante la implementación de programas de bienestar dirigidos al personal militar y civil y a sus familias, en los que el respeto y la promoción de los derechos humanos y aportes al desarrollo de la cadena aeronáutica y espacial permiten la articulación en un Programa de Sostenibilidad y Responsabilidad Social. Se concluye cómo la FAC evidencia gestión RS en comunión con los referentes internacionales en esta materia, como el Pacto Global, el Global Reporting Initiative (GRI) y la Organización para la Cooperación y el Desarrollo Económico (OCDE).

Palabras clave: buen gobierno, poder aéreo, rendición de cuentas, grupos de interés.

Resumo: $O$ presente artigo aborda a realidade na responsabilidade social dentro na Força Aérea Colombiana a partir do análise da estratégia, eu modelo e as praticas de sustentabilidade empreendida pela instituição que nas suas operações aéreas e outras capacidades distintivas ou habilidades especiais, define e aplica políticas do comando para promover a transparência e equidade entre outros valores. Com a implementação dos programas de bem-estar destinados o pessoal militar e civil e as suas famílias, nos que o respeito e a promoção dos direitos humanos e das contribuições para o desenvolvimento da cadeia aeronáutica e espacial permitem a articulação de um Programa de sustentabilidade e responsabilidade social. Conclui-se como na FAC evidência de gestão RS em comunhão com referências internacionais nesta materia como o Pacto Global, - Global Reporting Initiative (GRI) e da Organização para a Cooperação e Desenvolvimento Económico (OCDE)

Palavras-chave: Boa governação, Poder aéreo, Rendição de contas, Grupo do interesse

Abstract: This article discusses the reality of Social Responsibility (SR) within the Colombian Air Force from examination of the strategy, model and sustainability practices undertaken by the institution that, in its air operations and other distinctive capabilities or special skills, define and apply policies Command for promoting transparency and equity among other values. By implementing welfare programs aimed to military and civilian personnel and their families based on respect and promotion of human rights and contributions to the development of the aerospace chain allow joint in a Program Sustainability and Social Responsibility, it is concluded how the RS evidence FAC management in communion with the international benchmarks in this area as the Global Compact, the Global Reporting Initiative (GRI) and the Organisation for Economic Co-operation and Development (OECD).

Key Words: Accountability, Air Power, Good Government, Interest Groups. 


\section{Introducción}

Preguntarse al interior de una institución como la Fuerza Aérea Colombiana «ipara qué y por qué la Responsabilidad Social?» entraña entender que la legitimidad trasciende la gobernabilidad en tanto afianza el sentido del compromiso de sus miembros con el país, más allá de administrar las decisiones trazables del poder aéreo.

Conforme al cumplimiento de la misión institucional, hoy las unidades aéreas, en distintos puntos geográficos del planeta, no se desentienden del cambio de la cultura organizacional y, justamente por esta visión, la Fuerza Aérea Colombiana, consciente de su papel protagónico entre las Fuerzas del Aire del hemisferio y del mundo', es socialmente responsable ya que contribuye a la sostenibilidad de la sociedad y del entorno. Teniendo como referente otros casos de organizaciones militares y de policía que realizan actividades afines a esta dinámica, cabe destacar cómo las jornadas de apoyo al desarrollo, la extinción de incendios, la vigilancia vulcanológica, el transporte humanitario de personas y carga, la minimización de los impactos ambientales negativos y la creación de valor ambiental, contribuyen a la generación de espacios con sentido de pertenencia e impacto económico, social y ambiental.

En efecto, desde un comportamiento transparente y ético, que toma en cuenta las expectativas de sus partes interesadas para el cumplimiento de la legislación aplicable, es posible hablar de una Fuerza Aérea coherente con la normativa internacional de comportamiento, articulada al objetivo estratégico de fortalecimiento de la legitimidad institucional, bajo el entendimiento moral de buscar réditos sociales que le permitan su operación, crecimiento y perdurabilidad en el tiempo. En el caso de la Fuerza Aérea Colombiana, la motivación principal es el fortalecimiento de la legitimidad institucional, lo cual, a su vez, incide en el cumplimiento de la misión, en su capacidad de transformación y fortalecimiento para épocas de paz o conflicto, en asignaciones presupuestales, en la interacción con otros actores, en el bienestar de los integrantes de la Institución y en la suscripción y buen desarrollo de convenios de cooperación internacional, entre otros.

\section{Conceptualización y contexto}

Comprender la naturaleza y el alcance de la Responsabilidad Social -en adelante RS- al interior de la Fuerza

\footnotetext{
1. En 2013, la FAC presenta el Primer Reporte de Sostenibilidad y Responsabilidad Social, el cual se convierte en un referente mundial por ser la primera Fuerza Armada en irradiar, como organización, su tarea de sostenibilidad a manera de soporte angular de su quehacer visionario (Cfr. Pacto Global Colombia).
}

Aérea, implica examinar previamente conceptos asociados a la responsabilidad, como sostenibilidad, grupos de interés, diálogo social, estándares de comportamiento organizacional, mínimos legales, motivaciones de las organizaciones para ser socialmente responsables y medición y reporte de la responsabilidad.

Lo anterior, en razón de los vectores con los que la Norma ISO² entiende el modelo de gestión:

[E]l desarrollo sostenible se refiere a la integración de las metas de una calidad de vida elevada, la salud y la prosperidad con justicia social y al mantenimiento de la capacidad de la tierra para conservar la vida en toda su diversidad. Estas metas sociales, económicas y ambientales son interdependientes yse refuerzan mutuamente (ISO 26000, 2010, p. 17). ${ }^{3}$

Pero igualmente, desde ese hilo conductor se tiene que contextualizar la misión y la visión corporativa e institucional para responder a las exigencias del nivel de gestión que tienden a generar desarrollo y fortalecer la seguridad en los grupos sociales.

Hoy en día existe un consenso global sobre el papel y la responsabilidad de las empresas en el desarrollo de los países y de las regiones. Para los gobiernos, el desarrollo no se puede dar a cualquier precio, y por eso han elevado sus exigencias en materia de Responsabilidad Social y Sostenibilidad. No basta con que las empresas declaren voluntariamente sus acciones en estos aspectos, pues en muchos casos esta discrecionalidad se convierte en una herramienta publicitaria que no da cuenta sobre los impactos, reales o potenciales, negativos de sus operaciones, ni sobre la forma como se asume esa responsabilidad. En ese sentido, los gobiernos han decidido intervenir para favorecer una mayor transparencia y una mejor rendición de cuentas a los actores que intervienen en los diferentes mercados, a través de la exigencia de reportes de sostenibilidad

Por lo anterior, y fiel a su compromiso constitucional que en el Artículo 2 establece que son fines esenciales del Estado:

\footnotetext{
2. International Standarization Organization, entidad con sede en Ginebra (Suiza), que para el favorecimiento de normas, en especial para el tema que compete a procesos sostenibles como expresión de RS, concibe en la gestión medioambiental un preámbulo con la norma (ISO 14000) (Cfr. http://www.iso.org/iso/iso catalogue/catalogue tc/catalogue detail.htm?csnumber=46486).

3. En 2004, ISO celebró una conferencia internacional de múltiples partes interesadas sobre si se debería o no lanzar el trabajo en RS. La recomendación positiva de esta conferencia dio lugar a la creación, a finales de 2004, del Grupo de Trabajo de ISO sobre Responsabilidad Social (ISO/WG SR) para desarrollar la futura norma ISO 26000 (Cfr. Guía sobre Responsabilidad Social ISO 26000).
} 
Servir a la comunidad, promover la prosperidad General y garantizar la efectividad de los principios, derechos y deberes consagrados en la Constitución; facilitar la participación de todos en las decisiones que los afectan y en la vida económica, política, administrativa y cultural de la Nación; Defender la independencia nacional, mantener la integridad territorial y asegurar la convivencia pacífica y un orden justo (énfasis añadido) (Constitución Política de Colombia, Título 1, Artículo 2, p.1)

La Fuerza Aérea entiende que, en su misión de proteger el espacio aéreo nacional, su compromiso no se extiende únicamente a la protección ambiental como beneficio global sino que la dimensión social es una exigencia hacia la cual debe proyectarse para el logro del bienestar colectivo.

En esa dimensión resulta oportuno validar los conceptos de sostenibilidad y RS, para luego dimensionarlos dentro de la misión, visión y ejercicio de la Fuerza Aérea Colombiana.

\section{Concepto de sostenibilidad}

En la diversidad conceptual respecto a la sostenibilidad, para efectos del objetivo propuesto en este artículo, conviene referir las que con mayor énfasis se aplican institucional y corporativamente.

\section{El Informe Brundtland}

Dentro de este mandato es importante revisar el concepto de sostenibilidad que se deriva de la visión de desarrollo sostenible, introducida por primera vez en el Informe Brundtland en 1987, originalmente denominado Nuestro Futuro Común y elaborado para la ONU por una comisión que representaba a varias naciones, liderada por la primera ministra de Noruega Gro Harlem Brundtland (Ver Recuadro 1). Según Goodland et. àl. (1994, pp. 14-17), en ese entonces el desarrollo sostenible estaba asociado al medio ambiente y se definió como «el desarrollo que permite satisfacer las necesidades de la actual generación sin sacrificar la capacidad de las futuras generaciones para satisfacer sus propias necesidades» (Brundtland citado por Goodland et. àl., 1994, p. 15).

Montes (2007, p.16) insiste en que tanto los principios como los temas abordados por el Informe Brundtland ofrecen la posibilidad de extenderlos a otros ámbitos que trascienden los ecosistemas y que tienen que ver más con el impacto en proyección social.

\section{MANDATOS DE LA COMISIÓN MUNDIAL DE MEDIO AMBIENTE Y DESARROLLO DE NACIONES UNIDAS (1987)}

1. Examinar los temas críticos de desarrollo y medio ambiente y formular propuestas realistas al respecto.

2. Proponer nuevas formas de cooperación internacional capaces de influir en la formulación de las políticas sobre temas de desarrollo y medio ambiente con el fin de obtener los cambios requeridos.

3. Promover los niveles de comprensión y compromiso de individuos, organizaciones, empresas, institutos y gobiernos.

\section{TEMAS}

- Población y recursos humanos

- Alimentación

- Especies y ecosistemas

- Energía

- Industria

- El reto urbano

Recuadro 1. Aspectos puntuales del Informe Brundtland.

Fuente: Copyright (1994) por Goodland et. àl.

\section{Organización Internacional de Normalización ISO}

En la normatización ISO, el concepto de sostenibilidad también se aplica a las dimensiones social y económica (ISO 26000, 2010, p. 14). En esa medida, como existe una relación entre sostenibilidad social y concepto organizacional, es decir, si el entorno y la sociedad dejan de ser sostenibles, la organización no tiene un lugar para desarrollar sus actividades y no cuenta con personas con las que pueda interactuar para cumplir con la misión para la que fue creada. De ahí que una organización pueda contribuir con la sostenibilidad general o ir en contra de ella.

Precisamente, por esta lógica, las organizaciones buscan su permanencia en el tiempo y saben que esto depende de que sus grupos de interés se lo permitan y/o faciliten, lo cual se da en la medida que sus expectativas se satisfagan y que los impactos de la organización sean positivos o compensados cuando son negativos (Perdiguero y Reche, 2005, p. 61). En el caso específico de la Fuerza Aérea Colombiana, si el país o las regiones no son sostenibles, se compromete la perdurabilidad de la Institución, y si la Fuerza Aérea no contribuye con la sostenibilidad, su legiti- 
midad se debilita y los grupos de interés dificultarán o no apoyarán la operación.

\section{Concepto de responsabilidad social}

La primera referencia formal al concepto de responsabilidad aparece en 1889, en el libro El evangelio de la Riqueza de Andrew Carnegie, empresario y filántropo estadounidense (Stoner, 1996). Figura desde su concepto un enfoque filantrópico a la RS y se destaca el rol que pueden tener las empresas respecto a la atención de necesidades o de problemáticas sociales. Desde entonces, el concepto ha evolucionado pasando de ese enfoque filantrópico a uno estratégico, en donde no se trata de caridad corporativa, sino de obligación moral y de estrategia organizacional y social.

Asimismo, se nutre por convenciones como la Declaración Universal de los Derechos Humanos, la Declaración de la OIT sobre Principios y Derechos Fundamentales en el Trabajo, la Declaración de Río sobre el Medio Ambiente, la Agencia $21^{4}$, la Declaración de Copenhague y muchos planteamientos de diferentes autores, investigadores y referentes (Gaete, 2006, p. 132).

Para ilustrar el significado de RS se presentan algunas definiciones de organismos y referentes reconocidos, desglosados a continuación.

\section{Pacto Global (2004) \\ El Pacto Global (2004) señala que}

Ser socialmente responsable implica todo un modo de hacer negocios, que se manifiesta a través de las relaciones con la comunidad, el trato con los empleados y el respeto al medio ambiente. El respeto a estos principios hace a las empresas más competitivas, pues así contribuyen a generar un ambiente de negocios favorable para la inversión, el desarrollo económico y la modernización del país.

\section{ISO 26000 (2010)}

La responsabilidad se concibe como «el compromiso de una organización ante los impactos que sus decisiones y actividades ocasionan en la sociedad y el medioambiente, a través de un comportamiento transparente y ético» (ISO 26000, 2010).

\footnotetext{
4. Suscrita por 172 países miembro de Naciones Unidas, la Agenda 21 es producto de la Conferencia Mundial sobre el Medio Ambiente y Desarrollo Sostenible organizada por Naciones Unidas en Río de Janeiro (Brasil) en 1992, a manera de estrategia global para construir un modelo de desarrollo sostenible para el siglo XXI.
}

World Bussiness Council for Sustainable Development (WBCSD)

Esta organización global para el futuro sostenible de los negocios afirma que es el «compromiso que asume una empresa para contribuir al desarrollo económico sostenible por medio de la colaboración con sus empleados, sus familias, la comunidad local y la sociedad en pleno, con el objeto de mejorar la calidad de vida» (WBCSD, sf).

Icontec

El Instituto Colombiano de Normas Técnicas conceptúa que la responsabilidad social

es el compromiso voluntario que las organizaciones asumen frente a las expectativas concertadas que en materia de desarrollo humano integral se generan con las partes interesadas y que, partiendo del cumplimiento de las disposiciones legales, le permite a las organizaciones asegurar el crecimiento económico, el desarrollo social y el equilibrio ecológico (Icontec, sf)

Al recopilar conceptos se puede plantear que la RS:

es un comportamiento de la organización frente a sus grupos de interés; en un entendimiento más amplio y práctico, la RS hace referencia al mejoramiento de los impactos organizacionales cumpliendo con los mínimos legales y creando valor económico, social y ambiental a través de la contribución al desarrollo en las regiones o localidades en donde opera vía generación de empleo, contratación local, desarrollo de infraestructura, respeto y promoción de los derechos humanos, la salud, seguridad y bienestar para los trabajadores, uso racional de recursos, protección de la biodiversidad, mitigación o compensación de emisiones de CO2 y comportamiento ético y transparente (Daza, 2005, p. 263).

Al flexibilizar la concepción del talento humano dentro de la institución militar, es la toma de decisiones la que se enlaza de modo firme al compromiso compartido de mecanismos, procedimientos, objetivos y resultados; la FAC, en consecuencia, es sabedora de que de las ventajas competitivas depende en gran parte el que el enfoque no lineal del talento humano al interior de la Institución atienda a las fortalezas individuales y grupales, puesto que el direccionamiento estratégico conlleva a un primer acercamiento que indaga por la flexibilización de los procedimientos que la modernidad actual impone para un sector público perfilado a futuro y que, como el de la Fuerza Aérea, precisa de 
un replanteamiento que derive en la gerencia estratégica del proceso integral de la efectividad de cara a la RS, para hacerla sostenible.

\section{Concepto de grupos de interés}

Aunque en la propia definición de sostenibilidad y $R S$ se hace referencia al grupo de interés y se infiere su significado, conviene profundizar en esta categoría para facilitar la verificación de su aplicabilidad respecto a la Fuerza Aérea Colombiana; existen múltiples definiciones. Una definición aceptada en el mundo empresarial para grupo de interés o Stakeholders, aplicable a cualquier organización es: «Persona, Grupo, o Entidad que es impactada e impacta directa o indirectamente en las actividades de negocio de la empresa y por lo general están compuestos por la comunidad, los empleados y sus familias, los accionistas, los clientes, los proveedores, etc...» (Canadian Business for Social Responsability, citado por Fumás, 2005, p. 8).

Según la caracterización de grupos de interés, realizada por la Fuerza Aérea Colombiana dentro de sus grupos de interés, se encuentran entre ellos: miembros activos (oficiales, suboficiales, personal civil), soldados, personal retirado, comunidad cercana a las Unidades Aéreas, sociedad, medios de comunicación, universidades y centros de investigación, organismos de control (Contraloría, Procuraduría), Presidencia, Congreso, ministerios (Defensa, Hacienda, Protección Social), otras Fuerzas (nacionales e internacionales), gobernaciones, alcaldías, y gremios, entre otros, que se describirán más adelante.

Desde una perspectiva estratégica, los grupos de interés deben priorizarse, entendiendo que algunos tienen mayor importancia relativa para la organización. Para la priorización existen enfoques como el de Mitchell, Agle y Wood (1997) basado en los atributos de poder, legitimidad y urgencia.

\section{ESTRATEGIA DE LA FUERZA AÉREA COLOM- BIANA Y RESPONSABILIDAD SOCIAL}

Dado que las organizaciones encuentran en el ejercicio de la RS motivaciones que van desde lo moral hasta lo estratégico, cabe destacar que para la Fuerza Aérea Colombiana ser socialmente responsable está relacionado con el cumplimiento de su misión constitucional y con el fortalecimiento de su legitimidad. Con sus prácticas de RS, busca el respaldo de sus grupos de interés, así como garantizar el desarrollo de la operación para cumplir con su misión y visión y ser sostenible en escenarios de paz o conflicto.
Actualmente, la Fuerza Aérea Colombiana cuenta con alto grado de legitimidad; sin embargo, se gestiona su fortalecimiento para enfrentar con éxito los diversos y complejos escenarios que incluyen variables como actores al margen de la ley con capacidad política, causas y movimientos sociales transnacionales, organismos y grupos con capacidad de incidir en la operación y el cambio de necesidades y expectativas de los grupos de interés (Flórez y Jassir, 2006, p. 14).

En esta línea, la RS tiene un sentido estratégico para la Fuerza Aérea y por esto mismo cuenta con el respaldo del Alto Mando y está incorporada en diferentes instrumentos de gestión como el Plan Estratégico Institucional, las Políticas de Comando, la Política de Calidad y el Cuadro de Mando Integral. Adicionalmente, cuenta con un modelo y un Programa de Sostenibilidad y Responsabilidad Social que se estructura a partir de la oferta de valor y capacidades distintivas y de ocho ejes o pilares que serán descritos más adelante.

La Responsabilidad Social en la estrategia de la Fuerza Aérea Colombiana

La RS está vinculada con la estrategia de la Fuerza Aérea y se desarrolla a través de diferentes instrumentos de gestión. De acuerdo con el «Segundo Reporte de Sostenibilidad y Responsabilidad Social Institucional de la Fuerza Aérea Colombiana» (2015), los resultados en esta materia se ven reflejados en el fortalecimiento de la dinámica social, académica y económica del país. Bajo ese lineamiento, «realizar alianzas con instituciones del Estado, empresa privada y diferentes entidades y organizaciones locales, regionales e internacionales, hace parte de las acciones que realiza la Fuerza Aérea Colombiana para consolidar el territorio nacional e impulsar el desarrollo en diferentes lugares del país» (p. 2). Tal vinculación se evidencia con la formulación del objetivo estratégico no. 3: «Fortalecimiento de la Legitimidad Institucional».

Por otro lado, en el Plan Estratégico Institucional, PEI 2011 a 2030, específicamente en la estrategia no. 8 (PEI, 2011), se incluyen elementos de RS como el respeto por los derechos humanos y el Derecho Internacional Humanitario, la preservación del medio ambiente y el fortalecimiento de la Acción Integral para contribuir a la consolidación del Estado e impactar a la comunidad en diversos aspectos sociales y económicos. 
En la Figura 1 del Cuadro de Mando Integral de la Fuerza Aérea Colombiana se pueden observar diferentes elementos relacionados con la RS.



Figura 1. Mando Integral. Tomado del Plan Estratégico Institucional-PEl (2011-2030).

Fuente: www.fac.mil.co/mapa-estrat\%C3\%A9gico-institucional-2011-2030-0

Nótese que en la parte superior (círculo amarillo) está incorporado el objetivo n. 3 de Fortalecimiento de la Legitimidad Institucional y cómo en diferentes partes del Cuadro de Mando Integral se incluyen elementos relacionados con RS como las «Partes Interesadas» (Circulo Naranja) y «Preservar el Medio Ambiente» (Circulo Verde).

Cabe anotar que el Cuadro de Mando Integral sintetiza lo planteado en el Plan Estratégico Institucional - PEl, el cual se publica desde 1995 y se plantea como un instrumento que contribuye al cumplimiento de la visión y la misión en diferentes escenarios de paz o conflicto; además articula los diferentes frentes estratégicos y facilita la continuidad de las diferentes iniciativas estratégicas, a pesar de los posibles cambios de mando.

Otro instrumento de gestión en el que se vincula la RS es la Política de Calidad, en la cual se expresa que se pretende satisfacer las necesidades y expectativas de las partes interesadas (Flórez y Jassir, 2006, p. 23). 


\section{Política de la Calidad}

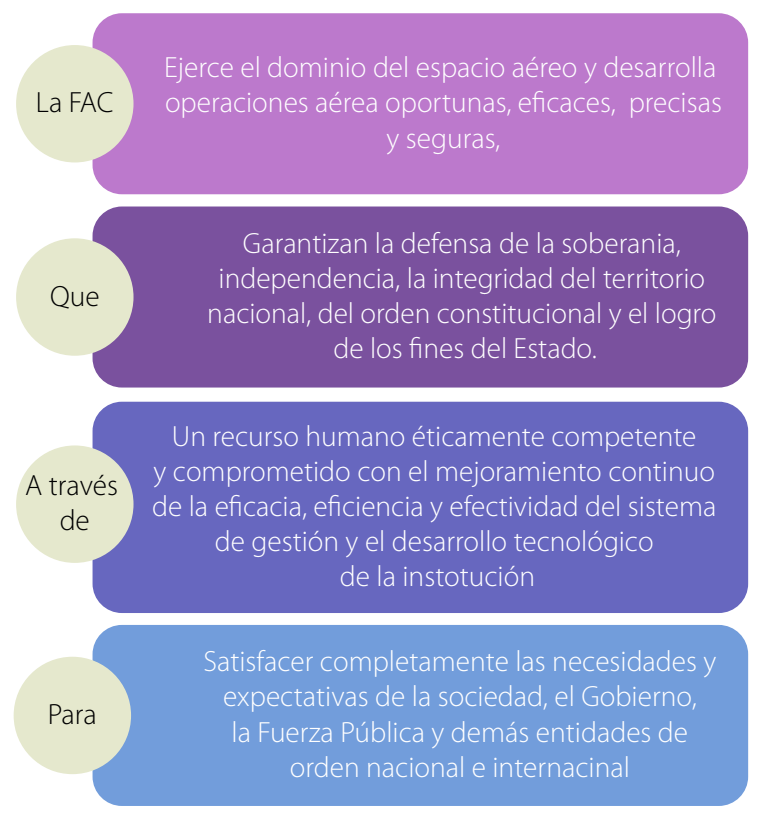

Figura 2. Política de Calidad de la Fuerza Aérea Colombiana.

Fuente: tomado del Departamento de Planeación Estratégica-Emape, Fuerza Aérea Colombiana (2005)

Nótese en la gráfica que el sentido de la misión, el quehacer y los recursos de la FAC están enfocados a satisfacer las necesidades y expectativas de un conjunto de partes interesadas o grupos de interés, elemento esencial dentro de la práctica de la RS.

Para que lo definido en la estrategia y en la política de calidad trascienda a lo operacional y táctico, el Alto Mando dispone de un conjunto de políticas, que basadas en el marco jurídico nacional, en los principios de Derechos Humanos y en el Derecho Internacional Humanitario, permean y orientan las visiones y misiones de los Procesos, Unidades Aéreas, Escuelas de Formación y demás dependencias que constituyen la estructura funcional de la entidad.

Impacto de la RS en la proyección sostenible de la Fuerza Aérea

Una Fuerza del Aire planeada a futuro sucumbe si no se anticipa a los retos que la defensa puede traer consigo, y si no se estructuran de forma articulada la tecnología, el talento humano y la interacción, cualquier comando aéreo, grupo de combate o de operaciones logísticas recae (López, 2002). De ahí que las matrices sobre las que se trazan nuevos mapas de planeamiento para la educación aeronáutica, apoyo logístico, seguridad y defensa de bases aéreas, encuentran un denominador común: la necesidad de suficiencia en la gestión de defensa aérea y adquiere entonces importancia el tornar sostenible la organización (p. 236).
Además de estas precisiones, se deduce de lo observado, tanto en las tareas de Aire de la Fuerza Nacional como en lo desarrollado desde el ángulo prospectivo por países como Inglaterra, Francia, Chile e Israel, que tanto en las amenazas presentes como futuras las Fuerzas Aéreas desempeñan desde ya un papel protagónico, por lo que fortalecer las capacidades de los hombres de la Fuerza Aérea que dominan el sistema de acción integral exige vincular directamente a la Responsabilidad Social para impactar con tareas de afianzamiento humano a las comunidades y grupos humanos asociados a su radio de acción (Drew, 2005, p. 19).

Así, el Comando de Defensa Aérea puede empoderar estrategias a futuro determinadas y, como Flórez y Jassir (2006) subrayan, la importancia de diseñar una estructura de prácticas de apoyo a los grupos sociales reside en que la nueva tendencia de la misión aérea es articular el poder aéreo con la promoción humana, «es ahí donde reside el sentido social y la visión sostenible de la Acción Integral, la Doctrina y la Política de Estado de la Fuerza Aérea Colombiana» (p. 37).

En efecto, cuando en el «Segundo Reporte de Sostenibilidad y Responsabilidad Social Institucional» (2015) se afirma que las cinco virtudes -justicia, prudencia, templanza, fortaleza y mística- que conforman la Fuerza Aérea contribuyen a que la doctrina de la Fuerza Aérea comulgue con el Modelo de Sostenibilidad y Responsabilidad Social Institucional, se destaca en consecuencia la mística como una de las cualidades con las cuales la Fuerza del Aire ha optimizado el desarrollo humano del país (p. 7). Estas tareas traducidas en las misiones emprendidas por sus miembros donde quiera que sus competencias se han puesto a prueba, reafirman lo expuesto por Hoffman (2002), que «la reingeniería del talento humano yace en poner las capacidades de la organización al servicio de los grupos de interés» (p. 212).

\section{Modelo de Responsabilidad Social de la Fuerza Aé- rea Colombiana \\ En armonía con su estrategia y modelo de gestión, la Fuerza Aérea Colombiana tiene un modelo de RS basado en su oferta de valor, capacidades distintivas y ocho ejes articulados para crear valor económico, social y ambiental. En la Gráfica 3 se muestra el modelo y sus componentes.}




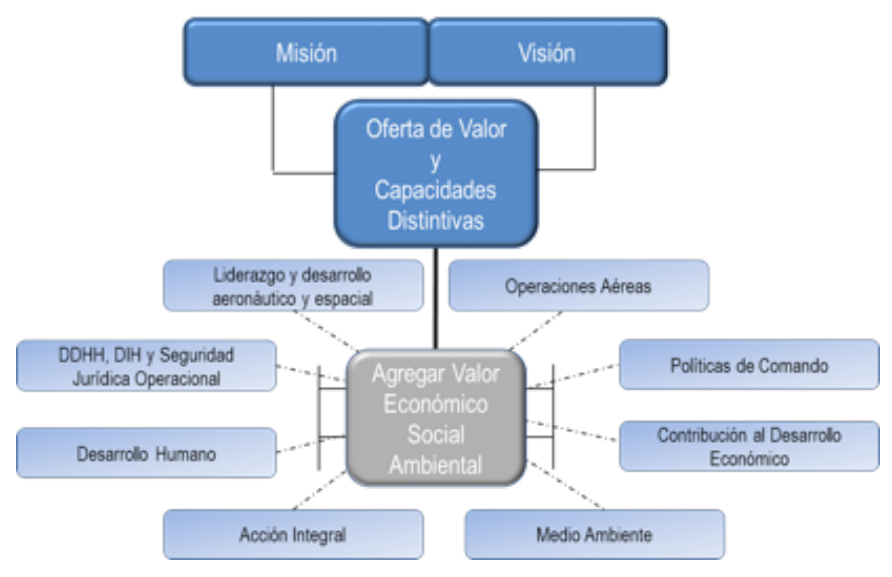

Figura 3. Modelo de Responsabilidad Social de la FAC (2013)

Nótese cómo la misión, la visión, la oferta de valor y las capacidades distintivas ocupan la parte superior del gráfico, como representación simbólica de que el ejercicio de RS está alineado con la estrategia y cimentado en sus características propias y diferenciadoras.

\section{Oferta de valor y capacidades distintivas}

La oferta de valor es el conjunto de beneficios que la FAC entrega a la sociedad y a los grupos de interés que la conforman, y las capacidades distintivas son las formas, recursos y habilidades de que dispone la Institución para entregar tal oferta de valor. A continuación, según Reporte RS de la FAC (2015), desde un enfoque de RS, se hace referencia a algunas de las capacidades de la FAC y a algunos de los beneficios que entrega a sus grupos de interés. ${ }^{5}$

- Transporte humanitario de pasajeros, suministros y equipo. Permite apoyar a los grupos de interés en diversos programas y actividades. Por ejemplo, se transportan personas, suministros y equipo para atender las Jornadas de Apoyo al Desarrollo que se realizan en diferentes lugares del país y los programas y actividades adelantadas en los municipios apadrinados por la Institución. También se contribuye con entidades territoriales como gobernaciones y alcaldías, ONG y empresas en desarrollo de convenios, con el intercambio de servicios o por solicitud de tales organizaciones y se realizan puentes aéreos para evitar el desabastecimiento y mantener la dinámica de poblaciones aisladas por razones de orden público como los bloqueos de carreteras (p. 5).

\footnotetext{
5. La oferta de valor y capacidades distintivas están descritas ampliamente en el PEI (pp. 18-29). La forma en que se presentan y describen en esta tesis son una adaptación desde una perspectiva de RS y no se incluyen las capacidades relacionadas con el cumplimiento de la misión en cuanto a vigilancia del espacio aéreo.
}

- Reacción eficaz ante emergencias o desastres. Se brinda apoyo ante emergencias y/o catástrofes. La participación de la Fuerza Aérea incluye prevención, como es el caso de la vigilancia vulcanológica y reacción como la extinción de incendios, rescate de personal y entrega de suministros y alimentos (p. 6).

- Recuperación de personal. Bajo la coordinación del Centro Nacional de Recuperación de Personal (CNRP) se brinda rescate o recuperación de personal militar en escenarios de conflicto y de personal civil en escenarios de accidentes y enfermedad en zonas de difícil acceso y/o desplazamiento (p. 6).

- Vigilancia energética y vial. Permite la protección de la infraestructura energética y vial del país. Favorece a toda la sociedad en tanto que se garantiza el suministro de energía y la movilidad para toda la comunidad y se protegen los activos de empresas públicas y privadas (p. 7).

- Operaciones de inteligencia. Permiten el diseño de los escenarios operacionales con el fin de medir y minimizar los impactos económicos, sociales y ambientales colaterales (p. 7).

- Apoyo al desarrollo económico y social del país. La FAC participa y en ocasiones lidera programas y actividades de desarrollo económico y social, como las Jornadas de Apoyo al Desarrollo. Por su reputación, ubicación geográfica y recursos de transporte aéreo tiene la capacidad de articular diferentes actores que suministran recursos que impactan económica y socialmente a diferentes comunidades y grupos de interés. Adicionalmente, a través de su vínculo con dos empresas comerciales del Estado adscritas al Ministerio de Defensa Nacional, impacta favorablemente a diferentes grupos de interés; con Satena (Servicio Aéreo a Territorios nacionales) se favorece el desarrollo de zonas alejadas como los antes llamados territorios nacionales y con la CIAC (Corporación de la Industria Aeronáutica Colombiana S.A) se genera empleo y se estimula el desarrollo de la cadena aeronáutica y espacial (p. 7).

- Compromiso con el control y la protección del medio ambiente. Además de cumplir con los mínimos legales, la FAC agrega valor ambiental a partir de la definición de zonas de reserva dentro de sus Unidades Aéreas, la denuncia ante las autoridades competentes de prácticas de minería ilegal, la contribución con las autoridades ambientales regionales y el desarrollo de proyec- 
tos especiales como Marandúa 7-4- 43, que entre otras cosas busca la compensación de emisiones a través de prácticas de reforestación (p. 8).

- Investigación y desarrollo aeronáutico. Contribuye al desarrollo de la cadena aeronáutica y espacial en tanto que realiza transferencia de tecnología a otros actores, genera empleo, realiza investigaciones e innovaciones que contribuyen a la sostenibilidad y crecimiento del país (p. 8).

- Es importante hacer notar que a las capacidades de la FAC y a los beneficios entregados a los diferentes grupos de interés se agrega el valor económico, social y ambiental, más allá de los mínimos que se derivan de la misión constitucional definida para la Institución.

\section{Ejes y prácticas de RS}

Desde su creación, la FAC ha contribuido al cumplimiento de los fines del Estado y ha impactado favorablemente al país a través de diferentes actividades y programas catalogados como de RS, en la medida que generan valor económico, social y ambiental a varios grupos de interés y contribuyen con la sostenibilidad propia y de la comunidad. Tales prácticas responden a la estrategia de la Institución, al contexto de sostenibilidad de las regiones y a las expectativas de los grupos de interés relacionados, y se articulan en un modelo de sostenibilidad y RS compuesto por ocho pilares que fueron definidos a partir de la realidad de la Institución, de su oferta de valor y capacidades distintivas y de los ámbitos, aspectos y componentes propuestos por diferentes referentes internacionales como el Pacto Global y la norma ISO 26000 anteriormente referenciada.

Pilares o ejes del modelo de sostenibilidad y RS de la Fuerza Aérea Colombiana

Operaciones aéreas

Son todas aquellas actividades que requieren el uso de aeronaves para su realización y que tienen impacto económico, social y/o ambiental en diferentes grupos de interés, por ejemplo: transporte humanitario de personal y carga, extinción de incendios, vigilancia energética y vial, puentes aéreos y rescate de personal, entre otros. No tienen equivalente en los referentes pero tienen relación con todos los ámbitos, por lo que bien podría llamarse un eje transversal de RS (FAC, 2011b, p. 34).

Cabe anotar que las operaciones aéreas que se realizan en cumplimiento de la misión y en favor de los intereses de la sociedad, y el uso legítimo de la fuerza, pueden generar impactos económicos, sociales y ambientales colaterales negativos, pero, incluso en estos escenarios, la FAC actúa con responsabilidad y en favor de la sostenibilidad, haciendo una planeación rigurosa de los escenarios operativos, aumentando la precisión del armamento y minimizando la letalidad.

\section{Políticas de comando}

Son parámetros de comportamiento organizacional fundamentados en normas superiores como la Constitución; leyes y decretos; principios internacionales como el derecho internacional humanitario; y normas propias, como valores y principios contenidos en el Código de Ética Militar Aérea:

Las Políticas de Comando equivalen al aspecto de Gobierno Corporativo definido para las empresas en los referentes de RS. Dentro de las Políticas de Comando están: aplicar el Código de Ética Militar Aérea, actuar con transparencia, eficiencia y austeridad en el gasto, acercarse a la comunidad, construir relaciones positivas con los grupos de interés, brindar atención al ciudadano, proteger al medio ambiente y contribuir con el desarrollo económico y social del país. Estas Políticas de Comando permiten que la estrategia de Responsabilidad Social descienda a los niveles operativos y tácticos de la organización y sea incorporada de manera efectiva en las Jefaturas, Departamentos, Unidades Aéreas, Escuelas y demás Dependencias que conforman la Institución (FAC, 2011a, p. 37).

Dentro de las prácticas adelantadas en este eje, se encuentran la aplicación de un «Código de Ética Militar Aérea» y un conjunto de principios y valores institucionales basados en la ética y en el bienestar general, entre otros que evitan cualquier tipo de discriminación en la incorporación y desarrollo de la vida laboral dentro de la Institución. La selección e incorporación no limitan a nadie por razones de credo, raza, ni género. La jerarquía está asociada al mérito y a la antigüedad.

\section{Contribución al desarrollo económico}

Este componente se relaciona con la capacidad que tiene la Fuerza Aérea para crear valor económico en su entorno y para sus grupos de interés, con la sostenibilidad financiera de la Institución y con la capacidad para poder responder económicamente a todas sus obligaciones.

Es importante tener en cuenta que la Fuerza Aérea es una entidad pública que no genera utilidades, así que es lógico que el valor recibido del presupuesto nacional sea retornado a la sociedad en forma de remuneración a los miembros de la Institución, pago de impuestos y el pago 
a proveedores; por ello, el mérito está en una ejecución eficiente y transparente como en efecto lo hace la Fuerza Aérea (FAC, 2011a, p. 34).

Más allá de los impactos económicos positivos que genera por el cumplimiento de su misión, por su lógico aporte como entidad pública y manejo adecuado de los recursos asignados, la Fuerza Aérea Colombiana, a partir de su oferta de valor y capacidades distintivas, entrega valor económico a la comunidad nacional e internacional. Por ejemplo, las actividades de la Fuerza Aérea en extinción de incendios, vigilancia del patrimonio minero y ambiental, transporte de carga y de pasajeros con fines sociales $y$ humanitarios y Jornadas de Acción Integral, favorecen la economía de las regiones e impactan favorablemente a grupos como las entidades territoriales, empresas privadas y la comunidad (Camargo, 2013, p. 28).

\section{Ambiental}

Este eje o pilar hace referencia al cumplimiento de los mínimos ambientales, la compensación de impactos negativos y la creación de valor ambiental. De acuerdo con sus políticas, existe un compromiso con el control y la protección del medio ambiente específicamente en desarroIlo del objetivo no. 12 de la Institución: «Preservar el medio ambiente: uso racional, conservación y protección de los recursos naturales, así mismo el manejo integral de los residuos generados al interior de las Unidades, enmarcado en la normatividad ambiental vigente. En general crear una cultura de respeto y conservación del medio ambiente» (FAC, 2010, Directiva permanente no. 18).

Como resume Ortega (2009), dentro de las prácticas ambientales de la Fuerza Aérea Colombiana se encuentran «la existencia en las Unidades Aéreas y Escuelas de Formación de un plan de manejo ambiental que se actualiza cada año para la sensibilización y educación ambiental a los Integrantes de la Institución y a la comunidad» (p. 27).

\section{Acción integral}

Se entiende como el conjunto de políticas, programas y actividades de integración con la comunidad y de colaboración en favor de ella. A través de la acción integral se pueden generar impactos sociales, económicos y/o ambientales en diversos grupos de interés, en especial sobre la comunidad. Dentro de este eje se incluyen programas como Plan Corazón Amigo, Piloto por un Día, Apadrinamiento de Municipios y Jornadas de Apoyo el Desarrollo. El concepto de acción integral estuvo asociado al de RS, pero en la actualidad ya se tiene claro que esta última tiene más alcances y que es un elemento transversal en la Institución. El concepto de acción integral tiene amplia relación con el componente «Sociedad» incluido en los referentes de responsabilidad social.

\section{Desarrollo humano}

Se refiere al bienestar, seguridad operacional ${ }^{6}$, el clima y la cultura organizacional y respeto y promoción de los derechos del personal militar y civil que integran la Fuerza Aérea Colombiana. Es el equivalente al aspecto laboral definido por los referentes internacionales, aunque es importante tener en cuenta que hay diferencias entre el personal civil y el militar y que algunos componentes, como la capacidad de asociación, no son aplicables. Algunas de las prácticas incluidas en este pilar se extienden a los familiares de los miembros de la Institución y también se desarrollan prácticas en favor de los retirados.

A continuación se relacionan algunas de las actividades de Desarrollo Humano que se adelantan en la FAC:

- Se asegura el cumplimiento de las condiciones de trabajo exigidas legalmente, incluido el salario adecuado, jornada laboral, vacaciones, licencias de maternidad y bienestar social.

- Se cuenta con mecanismos para asegurar la diversidad e igualdad de oportunidades de los integrantes de la Institución. Los ascensos están asociados al mérito.

- Se promocionan actividades de bienestar para el personal de la Fuerza Aérea y sus familias, a través de las campañas Somos la Fuerza y Mi Familia, Mi Fuerza. Se incluye promoción del deporte y la cultura física, así como la gestión del clima y la cultura organizacional -incluye la medición del clima, evaluación del personal, la evaluación de superiores y la promoción de los valores y principios institucionales, y realización de actividades para la integración familiar, entre otros-.

- Capacitación y actualización profesional. Los miembros de la Institución son educados (formados, capacitados y entrenados) permanentemente. Respecto a los soldados, son formados en cuatro especialidades: Seguridad Física Aeroportuaria; Seguridad y Control Aeroportuaria; Manejador Canino y Bombero Aeroportuario. Además, se capacitan en diversos temas para cuando regresen a la vida civil, e incluso algunos terminan sus últimos años del bachillerato mientras prestan su servicio militar obligatorio.

6. Seguridad Operacional es el equivalente al concepto de Seguridad Industrial, usado en el ámbito empresarial, y tiene relación con los riesgos laborales o profesionales. 
- Se toman acciones para selección y vinculación del personal, acordes con las exigencias legales, evitando situaciones de discriminación mediante un comité de antidiscriminación (Ortega, 2009, pp. 37-43).

\section{DD.HH., DIH y Seguridad jurídica operacional de la Fuerza Aérea}

Este eje cuenta con dos componentes. El primero se refiere al respeto y la promoción de los derechos humanos y el derecho internacional humanitario (DIH). Todas las operaciones aéreas se realizan con la premisa de respetar el DIH. La Fuerza Aérea Colombiana es pionera y líder en la gestión y desempeño de los derechos humanos y el derecho internacional humanitario. Tiene políticas y procedimientos que corresponden a la normatividad nacional y a estándares internacionales; sensibiliza, capacita y promociona los derechos humanos, y contribuye a que otras fuerzas aéreas, incluso de otros países, desarrollen sus capacidades en esta materia. Uno de los elementos diferenciadores de la Fuerza Aérea en DD.HH. y DIH es la existencia del ASEJO o Asesor Jurídico Operacional y la subordinación de todas las operaciones a la aprobación de este asesor.

Algunas de las prácticas en derechos humanos y derecho internacional humanitario de la Fuerza Aérea consisten en sensibilizar y capacitar a sus integrantes en derechos humanos y derecho internacional humanitario. Además, implementa programas de formación a todo nivel para integrantes de la Institución sobre los aspectos de derechos humanos relevantes para sus actividades (Camargo, 2013, p. 14).

\section{Liderazgo aeronáutico y espacial}

Desde la propuesta de los referentes internacionales de RS, particularmente desde las políticas de la OCDE, este aspecto consiste en el respeto a la propiedad industrial de terceros y en el alineamiento de los esfuerzos en ciencia y tecnología con las políticas que en esta materia tiene el país. Para el caso de la FAC, se incluye como un eje porque a través de las actividades relacionadas se generan impactos económicos, sociales y ambientales y se favorece la sostenibilidad de la Institución y de diferentes grupos de interés.

Al respecto cabe decir que la Fuerza Aérea tiene capacidades y prácticas de investigación, desarrollo e innovación que le permiten ejercer un liderazgo aeronáutico y espacial, contribuir con el desarrollo y dinamización de la industria y aportar en términos económicos, sociales e incluso ambientales. La Fuerza Aérea, a través de sus escuelas, realiza investigaciones aplicadas que contribuyen al desarrollo de la cadena aeronáutica y espacial; un ejemplo es EMAVI, la escuela de oficiales, que tiene líneas de investigación como Transporte de Carga Aeroespacial, Propulsión,
Materiales y Estructuras, Envejecimiento de Aeronaves, Diseño de Aeronaves Militares y Robótica Aérea. También participa en y lidera clústeres aeronáuticos convocando a universidades y empresas que permiten el avance de la tecnología, la ciencia y la investigación aeronáutica y espacial en beneficio del país (FAC, p. 17).

\section{Grupos de interés de la Fuerza Aérea Colombiana}

La Fuerza Aérea Colombiana se relaciona con gran cantidad de grupos de interés. Dentro de ellos se encuentran los siguientes:

- Integrantes de la Fuerza Aérea: Oficiales, suboficiales, personal civil y soldados. Reciben de la Fuerza Aérea desarrollo humano y profesional (capacitación), salud, seguridad jurídica y laboral.

- Fuerza Pública, nacional e Internacional: Compuesta por el Ejército Nacional, la Armada y la Policía, así como por fuerzas de otros Países. Tienen un vínculo de colaboración con la FAC. Reciben de la Fuerza una ventaja para el cumplimento de su misión y multiplicación de sus capacidades.

- Vinculados indirectos: Retirados y familiares de los integrantes de la Institución. Reciben beneficios como salud, recreación y capacitación por haber pertenecido a la Fuerza Aérea o por ser familiares de sus integrantes.

- Aliados estratégicos: Hace referencia a actores de industria aeronáutica (empresas, universidades, centros de investigación), oficiales de la reserva, ONG, entidades del Estado con servicios relacionados con las capacidades de la Fuerza (Ideam, lgac) y la Iglesia.

- Organismos de control y administración: Compuestos por actores del sistema legal (Contraloría, Procuraduría, Fiscalía, Defensoría), corporaciones autónomas regionales, ministerios y órganos de presupuesto y contratación.

- Representantes del poder Público: Entes del poder Ejecutivo, Legislativo y Judicial. La Fuerza le permite a este grupo el mantenimiento del orden público, la seguridad, la vigilancia de la infraestructura y, en general, el control de la soberanía del espacio aéreo.

- Comunidad: Compuesta por la sociedad en su conjunto.

- Organizaciones no gubernamentales: Organismos de socorro nacionales e internacionales como la Defensa Civil y la Cruz Roja. 
CIENCIA Y PODER AÉREO | ISSN 1909-7050 | E-ISSN 2389-9468 | Vol. 12 | Ene - Dic 2017 | Escuela de Postgrados de la Fuerza Aérea Colombiana | pp $176-190$

- Proveedores: Todas aquellas personas y organizaciones que entregan bienes o servicios a la Fuerza. Se dividen en proveedores operacionales, como los de aeronaves, equipos y piezas relacionadas y armamento, que son altamente especializados y tienen mayor poder o capacidad de incidencia; y los de apoyo, que son diversos, abundantes y con menor o nula capacidad de afectación sobre la Fuerza.

- Empresas y entidades beneficiarias: Gobernaciones, alcaldías, beneficiarios de transporte especial de pasajeros y empresas que reciben servicios, como el caso de Ecopetrol que es beneficiada con las misiones de vigilancia energética que realiza la Fuerza Aérea.

- Medios de comunicación: Locales, regionales, nacionales e internacionales. Son importantes debido al nivel de incidencia en la reputación de la organización por el manejo y divulgación de la información, referente a las operaciones y acciones de la Fuerza Aérea, que se suministre (FAC, 2014).

\section{Conclusiones}

La Fuerza Aérea es un actor social, por eso no puede estar ajeno a las expectativas de la sociedad. Aunque los impactos negativos pueden estar legitimados por la búsqueda del bien común, la Fuerza Aérea procura mejorarlos buscando la legitimidad.

En consecuencia, en la flexibilización de la gestión moderna al interior de las fuerzas del aire del mundo, el hecho de ser la FAC la primera fuerza aérea en presentar dos reportes serios, sustentables, sólidos y de resultados frente a la responsabilidad social en el mundo, su enfoque de la gestión sostenible se ha convertido en modelo para promover los equipos de alto desempeño dentro de la misión y visión del poder aéreo.

La FAC, como entidad pública que avizora el derecho internacional humanitario dentro del marco integral por el respeto a los derechos humanos y al medioambiente, tiene claras las reglas de la competitividad moderna, el desarrollo de los conceptos asociados a lo sostenible y a la responsabilidad social, cifrando su ejercicio en derroteros de eficiencia y prospectiva, pues en la medida en que planea el futuro de la Defensa Aérea, sus miembros, como cuerpo organizacional, optimizan sus capacidades como Fuerza Aérea en función de la administración y la promoción humana.

Por esta razón, al abordar la realidad de la responsabilidad social (RS) al interior de la Fuerza Aérea Colombiana, a partir del examen de la estrategia, el modelo y las prácticas de sostenibilidad emprendidas por la Institución, se evi- dencia su gestión RS en comunión con los referentes internacionales en esta materia, como el Pacto Global, el Global Reporting Initiative (GRI) y la Organización para la Cooperación y el Desarrollo Económico (OCDE). La resultante de esta aproximación da cuenta de que en sus operaciones aéreas y demás capacidades distintivas o habilidades especiales, como organización que vela por la defensa del espacio aéreo, define y aplica unas políticas de comando para la promoción de la transparencia y la equidad, acordes con las iniciativas internacionales en materia de responsabilidad social.

En virtud de lo anterior, sus programas de bienestar dirigidos al personal militar y civil y de sus familias, en los que el respeto y la promoción de los derechos humanos, y aportes al desarrollo de la cadena aeronáutica y espacial, permiten la articulación en un Programa de Sostenibilidad y Responsabilidad Social, que como parte de la Institución armada del país optimiza el liderazgo, canalizando la capacitación de su personal hacia el compromiso compartido con sus grupos de interés.

Para el logro de sus prácticas sostenibles, la toma de decisiones de la Fuerza Aérea se enlaza de modo firme al compromiso compartido de mecanismos, procedimientos, objetivos y resultados, esto es, en el direccionamiento de la Fuerza Aérea a la gerencia del Talento Humano para la reingeniería de recursos, desempeños, ejercicios y tareas conjuntas, de modo que lo operacional adquiera la dimensión debida en el conjunto de las apreciaciones de mando y los propósitos ejecutorios afines a lo sostenible y a la responsabilidad social que impone la exigencia global.

Desde esa óptica, la estructura de la organización es un medio que ayuda a la administración a alcanzar sus objetivos. Estos se extraen de la estrategia global de la empresa; se podría afirmar que su estrategia está alineada con la estructura de la organización, la sinergia de su Talento Humano y el curso normal operativo y de apoyo, originando niveles de satisfacción en los procesos de prioridad institucional. Estas razones han llevado a la Fuerza del Aire de Colombia a la formulación de correctivos, por lo que su compromiso con las decisiones y prácticas que impactan positivamente a los grupos humanos que ella promueve minimiza tiempos de espera y maximiza una comunicación más fluida y una definición muy exacta de las funciones e iniciativas de ayuda y de apoyo para alcanzar los resultados esperados, acordes con la estrategia de la Institución.

Finalmente, como la Fuerza Aérea tiene claro los conceptos de estrategia, estructura, desarrollo y cultura organizacional, y la interrelación de estos con los grupos 
de interés y la sociedad colombiana en general, dimensiona los niveles administrativos del Talento Humano en las Unidades que a diario deben manejar las necesidades y oportunidades que la Institución requiere para su desempeño frente a las múltiples exigencias de hoy. Su sentido de gestión, con sentido de oportunidad, beneficio y proyección, dentro de la idea organizacional de la Fuerza del Aire a futuro, le permite generar cultura corporativa con transparencia para ponderar sus ventajas competitivas sin la amenaza de quedar a la deriva por la duplicidad de procesos, puesto que con la vocación del Aire está llamada a la resolución de problemas serios de estructura y procesos, realidad que debe alinearse con el objetivo estratégico impuesto por la Institución.

Para finalizar, proyectarse corporativamente no implica únicamente mayor modernidad tecnológica sino una activación del recurso humano con sentido global.

\section{Referencias}

\section{Fuentes académicas}

Almonacid Carrasco, H. (2010). La Responsabilidad Social en las adquisiciones del ejército.Chile: Universidad de Chile

Camargo Abril, J. C. (2013). Diseño presentación y propuesta de implementación de una guía técnica para la administración y gestión eficaz de riesgo institucional en la Fuerza Aérea Colombiana basado en la NTC-ISO 31000: 2011 y buenas prácticas organizacionales con el Sistema de Gestión Integrado (Tesis doctoral), Escuela Colombiana de Ingeniería Julio Garavito, Bogotá.

Cheyre, J. E. (2008). La transformación del ejército chileno: un caso de análisis para América Latina. Foreign Affairs Latinoamérica, 8(3), 52-64.

Daza, E. F. (2005). Reflexiones en torno a la Responsabilidad Social de las Empresas, sus políticas de promoción y la economía social. CIRIEC-Espala, (53), 261-283.

De Solminihac, C. L. F. (2011). Responsabilidad social de las Fuerzas Armadas en tiempo de paz. FUERTE LESLEY J. McNAIR WASHINGTON, DC

Drew, C. D. M. (2005). Educando a los Oficiales de la Fuerza Aérea. Buenos Aires

Fumás, V. S. (2005). ¿Sustituye le responsabilidad social al buen gobierno de la empresa? Economistas, 23(106), 4-12.

Flórez, T. C. J. P. S., y Jassir, F. M. J. (2006). La Fuerza Aérea Colombiana y la Acción Integral, Doctrina y Política de Estado. Air \& Space Power Journal, 30-36

Gaete, R. (2006). La Universidad y la responsabilidad social. Revista de Estudios y Experiencias en Educación REXE 5(10), 129-145.
García, A. (2007). Responsabilidad Social Empresarial. Su contribución al desarrollo sostenible. Revista Futuros, 5, 17.

Goodland, R., Daly, H. E. H., Trygve Hansen, S. T., Jan Hueting, R. S., Salah El Droste, B. V. D., Peter Costanza, R. B., y Lester Postel, S. F. (1994). Desarrollo económico sostenible: avances sobre el Informe Brundtland (No. HD79 G65e).

Lechner, N., y Güell, P. (2006). Construcción social de las memorias en la transición chilena. Subjetividad y figuras de la memoria, Construcción social de las memorias en la transición chilena, 17-46.

López, J. A. L. (2002). El poder aéreo: instrumento decisivo para la resolución de las crisis del siglo XXI. Arbor: Ciencia, pensamiento y cultura, (674), 231-258.

Mitchell, R. K., Agle, B. R., y Wood, D. J. (1997). Toward a Theory of Stakeholder Identification and Salience: Defining the Principle of Who and What Really Counts. Academy of management review, 22(4), 853-886.

Montes, C. (2007). Del desarrollo sostenible a los servicios de los ecosistemas. Revista Ecosistemas, 16(3), 1-3

Núñez, G. (2003). La responsabilidad social corporativa en un marco de desarrollo sostenible. 72. United Nations Publications.

Ortega Ch., P.A. (2009). Historia de la Fuerza Aérea Colombiana FAC. Bogotá.

Perdiguero, T. G., y Reche, A. G. (Eds.). (2005). La responsabilidad social de las empresas y los nuevos desafíos de la gestión empresarial. España: Universidad de Valencia

Sánchez, R., Hoffmann, J., Micco, A. et al. (2003). Port Efficiency and International Trade: Port Efficiency as a Determinant of Military Cost. Maritime Economics \& Logistics, 5(2), 199-218.

Schvarstein, L. (2015). Inteligencia social de las organizaciones. Libros Editorial UNIMAR.

Stoner, J. (1996). Administración. México D.F.: Prentice Hall.

\section{Fuentes corporativas e institucionales}

FAC Fuerza Aérea Colombiana. (2011a). Plan Estratégico Institucional 2011-2030. Bogotá: Imprenta y Publicaciones Militares

. (2011b). Manual FAC 3-56. Manual de Requisitos y Procedimientos para tripulaciones de la Fuerza Aérea Colombiana. Bogotá: Imprenta y Publicaciones Militares.

(2014). Políticas de Comando. Políticas de Comando (Políticas de Operación). Bogotá: Imprenta y Publicaciones Militares.

(2015). Segundo Reporte de Sostenibilidad y Responsabilidad Social Institucional 2015. Bogotá: Imprenta y Publicaciones Militares.

International Standarization Organization. Norma, I. S. O. (2006). 
CIENCIA Y PODER AÉREO | ISSN 1909-7050 | E-ISSN 2389-9468 | Vol. 12 | Ene - Dic 2017 | Escuela de Postgrados de la Fuerza Aérea Colombiana | pp 176-190

26000: Guía sobre Responsabilidad Social. Boletín. Recuperado de https://www.iso.org/obp/ui\#iso:std:iso:26000:ed$1: \mathrm{v} 1: \mathrm{es}$

Ministerio de Defensa Nacional. (2008). Proyecto Educativo de las Fuerzas Armadas -PEFA-. Bogotá: Imprenta y Publicaciones Militares.

World Commission on Environment and Development. (1987).

Our common future. Oxford: Oxford University Press.

\section{Fuentes documentales}

Initiative, G. R. (2006). Guía para la elaboración de memorias de sostenibilidad. Ámsterdam: GRI.

\section{Fuentes jurídicas}

Directiva Permanente No 018 - 2010, Fortalecimiento Gestión Ambiental FAC que hacer

Directiva Permanente 800-4 del 14 de febrero de 2003: Mediante la cual, se formula el Plan Permanente de Integración del Derecho Internacional de los Derechos Humanos (DIDH) y del Derecho Internacional aplicable a los Conflictos Armados (DICA), en los manuales de doctrina operacional y formación militar.

Directiva Permanente 051 del 13 de junio de 2007: Mediante la cual se emite un plan de acción para implementar las recomendaciones del estudio sobre el sistema de enseñanza de DDHH y DICA.

Directiva permanente 50 del 10 de julio de 2007 y Circular 6590 del 27 de diciembre de 2007: Respeto al DIH y protección de la población civil.

Directiva permanente 300-19 del 06 de junio de 2007: Las Fuerzas Militares en cumplimiento de su misión deben brindar protección y apoyo a comunidades afrocolombianas, palenqueras y raizales en riesgo.

Directiva Permanente 048 del 28 de febrero de 2008: Protección a niños, niñas y adolescentes.

Directiva Permanente 5 del 21 de abril de 2010: Política de Gestión Ambiental para el Sector Seguridad y Defensa.

Directiva Permanente 046 del 30 de marzo de 2010: Lineamientos para la formulación de Planes de Acción Ambiental.

Directiva Permanente 167 del 01 de septiembre de 2009: Guía para el diseño e implementación del Sistema de Gestión Ambiental (SGA) para las Unidades Militares de las FF.MM. bajo NTC ISO 14001

Directiva Permanente 166 del 01 de septiembre de 2009: Fortalecimiento de la Gestión Ambiental de las Fuerzas Militares

República de Colombia. (1991). Constitución Política de Colombia. Título 1, Artículo 2 p.1.

\section{Fuentes electrónicas}

Pesce, D. (2015). El Espectador. Recuperado de http://www.elespectador.com/tomalapalabra/pacific-rubiales/la-politicapublica-se-mueve-del-reporte-240-articulo.

Departamento Nacional de Planeación. Plan Nacional de Desarrollo 2010-2014. Bogotá. Recuperado de www.dnp.gov.co/ PortalWeb/PND/PND20102014.aspx. P. 21

World Business Council for Sustainable Development. Ginebra. Recuperado de http://www.wbcsd.org/home.aspx.

ICONTEC internacional. (2016). Bogotá. Recuperado de http://www. icontec.org/index.php/es/guia-tecnica-colombiana-180-de-rs

Ministerio de Defensa. Gestión estratégica. Bogotá. Recuperado de https://www.mindefensa.gov.co/irj/go/km/docs/ documents/GSED/Documentos/Resultados/Gestion\%20 Estrat\%C3\%A9gica.pdf 
